\title{
Management and evaluation of treatment adherence and effectiveness in chronic venous disorders: results of the international study VEIN Act Program
}

\author{
Vadim Bogachev ${ }^{1}$. José Manuel Jiménez Arribas ${ }^{2} \cdot$ Sorin Baila $^{3} \cdot J^{\prime}$ Jorge Ulloa Dominguez ${ }^{4} \cdot$ Johannes Walter $^{5}$. \\ Dale Maharaj ${ }^{6} \cdot$ Andres Marin $^{7}$
}

Published online: 27 May 2019

(c) The Author(s) 2019

\begin{abstract}
Objective The aim of this study was to examine worldwide chronic venous disease (CVD) burden and evaluate effectiveness, adherence and satisfaction with conservative treatment.

Materials and methods The VEIN Act Program (VAP) was an international, prospective, observational study that enrolled adult outpatients with lower-limb pain consulting for any CVD-related clinical presentation. Patients' CVD symptoms, Clinical Etiological Anatomic Pathophysiologic (CEAP) classification, and symptom intensity were recorded. After prescribing conservative treatments, patients returned for a follow-up visit.

Result A total of 567 physicians enrolled 7987 patients, 7397 of whom were included in the analysis. Patients were mainly female (79.3\%), mean age was $54.0 \pm 15.1$ years and mean body mass index was $26.8 \pm 4.8 \mathrm{~kg} / \mathrm{m}^{2}$. CEAP clinical classifications were $\mathrm{C}_{0 \mathrm{~s}}(3.6 \%), \mathrm{C}_{1}(19.4 \%), \mathrm{C}_{2}(21.8 \%), \mathrm{C}_{3}(32.0 \%)$ and $\mathrm{C}_{4}-\mathrm{C}_{6}(23.2 \%)$. Symptoms were reported by $89.6 \%$ of patients and were present across all CEAP classes. Mean intensity $(10 \mathrm{~cm}$ visual analog scale) was $>5 \mathrm{~cm}$ for all symptoms. Only $30.7 \%$ of patients had previously consulted for leg problems, and $24.3 \%$ had previously received treatment. Conservative treatment alone was prescribed to $78.3 \%$ of patients, and in association with operative treatment in $21.6 \%$ of patients, and consisted mostly of venoactive drugs (VADs) [99.6\%, mostly micronized purified flavonoid fraction] and compression therapy (63.4\%) in combination with lifestyle changes such as weight loss. Adherence to prescribed duration was $65.2 \%$ for VADs, but only $29.1 \%$ wore compression therapy as prescribed. Symptom relief was high with VADs and compression therapy (>96\%), and the majority of patients were satisfied (94.1\%).
\end{abstract}

Conclusion Conservative treatments were beneficial across the spectrum of CVD, highlighting the importance of identifying patients early in the disease course.

Vadim Bogachev

Vadim.bogachev63@gmail.com

1 Pirogov Russian National Research Medical University, Sadovaya-Kudrinskaya str.28/30, app.6, 123001 Moscow, Russia

2 Clínica San Juan de Dios de Pamplona y Policlínica Navarra de Tudela, Tudela, Spain

3 Emergency Institute for Cardiovascular Diseases "Prof. Dr. C. C. Iliescu", Bucharest, Romania

4 Vascular Surgery Fundación Vascular de Colombia, Bogotá, Colombia

5 Private Practice, Salzburg, Austria

6 Caribbean Vascular and Vein Clinic, St. Clair Medical Center, Port of Spain, Trinidad and Tobago

7 Hospital Metropolitano de Santiago, Santiago, Dominican Republic

\section{Introduction}

The term chronic venous disease (CVD) covers a spectrum of clinical presentations of varying severity, from telangiectasies to active venous ulcers. Studies that have examined the prevalence of the individual manifestations indicate that the most common are telangiectasies, reticular veins, and varicose veins [1-3]. However, prevalence estimates for CVD in the literature vary widely because of differences in the methods of evaluation, criteria for definition, and the geographic regions analyzed, with higher prevalence being generally reported in developed countries [1]. Other factors to consider include variability in the distribution of CVD risk factors, the subjective nature of the condition in the early stages, physician accuracy in diagnosing the condition, and the quality and availability of diagnostic resources such 
as duplex ultrasound [1]. In addition, most survey data are obtained from patients who have consulted their physician and therefore do not capture the significant numbers of individuals who may have CVD but do not visit their physician.

A comprehensive review of the medical literature up to 2005 identified prevalence rates for varicose veins of $<1 \%$ to $73 \%$ in women and $2-56 \%$ in men [1]. In a nationwide US program among individuals invited for a free screening, physicians identified varicose veins in over $30 \%$ of 2234 participants, and more advanced CVD in over $10 \%$ [2]. A more recent European epidemiological study [3] used the revised CEAP (Clinical manifestations, Etiologic factors, Anatomic distribution of disease, and underlying Pathophysiology) classification to grade CVD of the lower extremities: $\mathrm{C}_{0 \mathrm{~s}}$ (venous leg symptoms); $\mathrm{C}_{1}$ (telangiectasias, reticular or spider veins); $\mathrm{C}_{2}$ (varicose veins); $\mathrm{C}_{3}$ (edema); $\mathrm{C}_{4}$ (skin changes); $\mathrm{C}_{5}$ (healed venous ulcers); and $\mathrm{C}_{6}$ (active venous ulcers) [4]. A total of 406 physicians screened 10-20 consecutive patients attending for a routine visit (total number $>6000$ ) [3]. The reported prevalence values for the individual CEAP clinical classes were: $\mathrm{C}_{0 \mathrm{~s}}(13.9 \%), \mathrm{C}_{1}(18.7 \%)$, $\mathrm{C}_{2}(16.7 \%), \mathrm{C}_{3}(13.7 \%), \mathrm{C}_{4}(9.2 \%), \mathrm{C}_{5}(2.1 \%)$, and $\mathrm{C}_{6}(0.9 \%)$ [3].

CVD is an important cause of discomfort and disability, and in many cases is progressive, with long-term consequences that may be serious for the patient [5]. Qualityof-life $(\mathrm{QoL})$ assessment is now routinely recommended for evaluating the disease burden for a patient [6] and has demonstrated that CVD has a negative impact on QoL at all stages, which worsens with more advanced disease [3]. This is related to a number of symptoms, including pain, leg heaviness, itching, and sensation of swelling; the psychological impact of unsightly legs; and the chronic nature of the disease and its treatment. There is also increasing recognition that CVD is a disorder of socioeconomic significance. Longer life expectancy worldwide, coupled with the fact that older age is a risk factor for CVD, mean that the prevalence of the condition will rise in future decades, with a subsequent impact on healthcare costs. Indirect costs related to loss of work productivity may also increase [7].

The high prevalence and broad spectrum of conditions that CVD encompasses, combined with its QoL and economic impact, highlight the need for well-tolerated and costeffective treatments that can reduce the presence of symptoms and their severity and, whenever possible, correct the underlying abnormality before progression to more severe stages occurs [8]. Conservative treatment, largely comprised of lifestyle advice, compression therapy and venoactive drugs (VADs), is effective for slowing or preventing progression of CVD in the lower CEAP classes, and for the relief of symptoms throughout the disease $[9,10]$; however, data are lacking on the effectiveness of such treatments in the broad range of CVD patients worldwide with varying CVD risk factors, stage of disease, and symptom severity. The VEIN Act Program (VAP) was an observational survey, carried out under the auspices of the European Venous Forum (EVF). It differs from other CVD observational studies in that it simultaneously collected large-scale data on the epidemiology of CVD with data on the use and effectiveness of conservative therapy in the framework of routine consultations. The survey examined CVD burden among populations in different regions of the world and evaluated benefit, adherence, and satisfaction with conservative treatment in a wide range of patients. The aim was to collect information that would improve CVD management strategies. The current paper will present the global results from the VAP.

\section{Methods}

The VAP was an international, prospective, observational study conducted between March 2013 and December 2016 in seven regions/countries (Russia, Spain, Romania, Colombia, Austria, West Indies and Central America) by a total of 567 physicians. Approval confirming compliance with the ethical standards laid down in the Helsinki Declaration amended in October 2008 was obtained from the Ethics Committees of the countries in which the program was implemented. Eligible patients were (1) aged $>18$ years; (2) visiting their practice with complaints of pain in the lower limbs or any clinical presentation related to CVD; and (3) not receiving ongoing treatment for CVD, although they may have been treated in the past. Patients consulting for an emergency or for an acute episode of an ongoing event were excluded, as were those with concomitant diseases or treatments that might interfere with lower limb pain or edema. Informed consent was obtained from each participant.

Each subject attended two visits at the investigation site. At the inclusion visit (V0), all patients underwent a clinical examination of the lower limbs to identify the presence and severity of CVD signs. A subject was diagnosed as suffering from CVD when any sign of venous pathology (CEAP clinical classification $\mathrm{C}_{1}-\mathrm{C}_{6}$ ) was seen in at least one leg, and/or any symptom, even if not associated with signs $\left(\mathrm{C}_{0 \mathrm{~s}}\right)$ [11]. Patients were asked to indicate the intensity of their symptoms with the use of a $10 \mathrm{~cm}$ visual analog scale (VAS). A CEAP clinical class was then assigned, taking into account the highest descriptor (for example, a patient with both telangiectasies and varicose veins was classified $\mathrm{C}_{2}$ ) [11]. Following confirmation of a CVD diagnosis, physicians completed a questionnaire detailing the patient's clinical presentation and history, presence of CVD signs and/or symptoms, and conservative treatment prescribed, listing all treatment characteristics. According to the physicians' usual practice, patients were prescribed conservative treatment for CVD and requested to return for a follow-up visit (V1) in 
accordance with routine clinical practice within 8 days of the end of the prescribed treatment. At the V1 visit, physicians completed a second questionnaire detailing whether the patients had adhered to the treatment, including information on whether they had been able to purchase the prescribed brand, and taken the dose as prescribed for the correct treatment duration, and, in the case of non-adherence, the reasons why (forgot to take medication, no drinking water available, lack of efficacy, too difficult to swallow, taking other pills).

For compression therapy it was noted whether patients wore the hosiery, as prescribed, most days, intermittently or not at all. If treatment was stopped or not taken as prescribed, the reasons for this were noted (too difficult to put on, unattractive, causes sweating, uncomfortable, causes skin problems/itching, and ineffective). The V1 questionnaire also collected information on the effectiveness of the prescribed treatment (presence/absence of symptoms, time of relief onset) and information on the signs and symptoms of CVD (CEAP clinical classification, VAS). At this visit patients were asked to score their satisfaction with treatment on a 5-point scale from (0) not satisfied at all; (1) rather unsatisfied; (2) rather satisfied; (3) very satisfied; and (4) extremely satisfied.

\section{Statistical analysis}

Quantitative data were represented by the number of patients $(n)$ and expressed as the mean value and standard deviation (SD); qualitative data were expressed as the number $(n)$ and percentage $(\%)$ of patients. Statistics were mainly descriptive and were performed in patients with complete data for main variables and no relevant deviations from the protocol. Adherence to conservative therapy was described in all patients from the analysis set. Patients with operative treatment were excluded from conservative treatment effectiveness analysis. The proportion of patients with symptoms was compared between V0 and V1 using Chi square tests, and the intensity of each symptom was compared between $\mathrm{V} 0$ and V1 using paired Student $t$ tests. All tests were two-sided, and a $p$ value $<0.01$ was considered statistically significant.

\section{Results}

A total of 567 physicians (223 general practitioners, 166 vascular surgeons, 84 phlebologists, 53 dermatologists, and 41 other) enrolled 7987 subjects to take part in the study from the following countries and regions: Colombia $(n=3030)$; Romania $(n=2496)$; Russia $(n=1607)$; Spain $(n=469)$; West Indies $(n=195)$; Central America $(n=169)$; and Austria $(n=21)$. Results from 485 subjects were excluded from the main analysis for reasons relating to missing data and not meeting the inclusion criteria, leading to 7502

\begin{tabular}{|c|c|}
\hline Characteristic & Results \\
\hline Male/female [no. of patients (\%)] & $1532(20.7) / 5865(79.3)$ \\
\hline Mean age $\pm S D$, years & $54.0 \pm 15.1$ \\
\hline \multicolumn{2}{|l|}{ Age groups, years [no. of patients (\%)] } \\
\hline $18-34$ & $887(12.0)$ \\
\hline $35-50$ & $2058(27.8)$ \\
\hline $51-64$ & $2572(34.8)$ \\
\hline$\geq 65$ & $1880(25.4)$ \\
\hline Mean $\mathrm{BMI} \pm \mathrm{SD}, \mathrm{kg} / \mathrm{m}^{2}$ & $26.84 \pm 4.82$ \\
\hline \multicolumn{2}{|l|}{ BMI groups, $\mathrm{kg} / \mathrm{m}^{2}$ [no. of patients (\%)] } \\
\hline$\leq 18$ & $72(1.0)$ \\
\hline $19-24$ & $2688(36.3)$ \\
\hline $25-29$ & $2979(40.3)$ \\
\hline$\geq 30$ & $1658(22.4)$ \\
\hline $\begin{array}{l}\text { Previous consultation for leg problems } \\
\text { [no. of patients (\%)] }\end{array}$ & $2268(30.7)$ \\
\hline $\begin{array}{l}\text { Previous treatment for leg problems } \\
\text { [no. of patients }(\%)]\end{array}$ & $1800(24.3)$ \\
\hline
\end{tabular}

$B M I$ body mass index, $S D$ standard deviation

eligible patients with complete data at V0 visit. Of these, 105 patients (1.4\%) who did not attend the $\mathrm{V} 1$ visit were also excluded from the analysis. The final analysis set therefore comprised 7397 patients. The mean time between visits V0 and V1 was 80 days $(\approx 2.5$ months).

\section{Patient characteristics}

Patients' baseline characteristics are shown in Table 1. The study population comprised 5865 women and 1532 men. A higher proportion of women (79.3\%) than men (20.7\%) consulted for CVD, although the proportion of men increased after the age of 65 years. Mean age was $54.0 \pm 15.1$ years, with the mean age in men being $\approx 2$ years greater than in women. The highest proportion of patients was in the 51-64 years age group $(34.8 \%)$. Mean body mass index (BMI) was $26.8 \pm 4.8 \mathrm{~kg} / \mathrm{m}^{2} ; 40.3 \%$ of patients were overweight and $22.4 \%$ were obese. Around one-third of subjects (30.7\%) had previously consulted for leg problems and onequarter of subjects $(24.3 \%)$ had previously received treatment. The most symptomatic patients were most likely to have had a prior consultation for leg problems.

As part of the survey, physicians were required to assign each subject to the appropriate severity stage of the CEAP clinical classification. Overall, 261 (3.5\%) subjects did not show any visible signs, but had symptoms only $\left(\mathrm{C}_{0 \mathrm{~s}}\right), 1411$ $(19.1 \%)$ had telangiectasies or reticular veins $\left(C_{1}\right), 1593$ (21.5\%) had varicose veins $\left(\mathrm{C}_{2}\right), 2380(32.2 \%)$ had edema $\left(\mathrm{C}_{3}\right), 796(10.8 \%)$ had pigmentation or eczema $\left(\mathrm{C}_{4 \mathrm{a}}\right), 406$ 


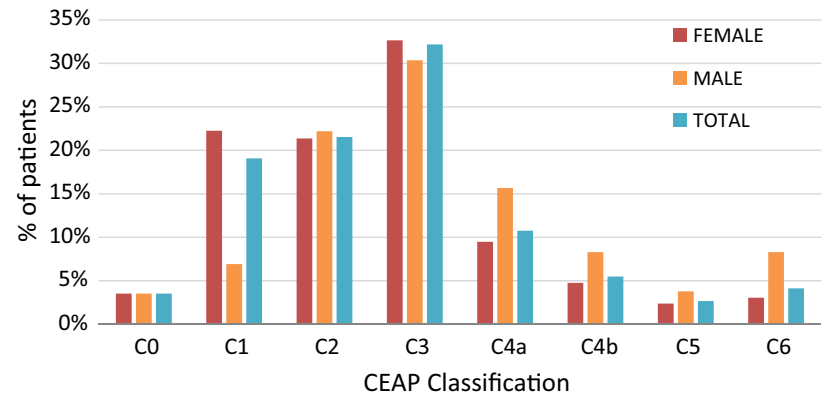

Fig. 1 Patients' CEAP classification at the inclusion visit (V0). CEAP Clinical Etiological Anatomic Pathophysiologic

(5.5\%) had lipodermatosclerosis or atrophie blanche $\left(\mathrm{C}_{4 \mathrm{~b}}\right)$, 198 (2.7\%) had healed venous ulcers $\left(\mathrm{C}_{5}\right)$, and 306 (4.1\%) had active venous ulcers $\left(\mathrm{C}_{6}\right)$ (Fig. 1). Women had a much higher prevalence of $\mathrm{C}_{0}-\mathrm{C}_{1}$ than men (25.8 vs $\left.10.4 \%\right)$, but this difference was not apparent in the $\mathrm{C}_{2}$ class. Men were more likely to suffer from the more severe reported CVD signs $\left(\mathrm{C}_{4 \mathrm{a}}, \mathrm{C}_{4 \mathrm{~b}}, \mathrm{C}_{5}\right.$ and $\left.\mathrm{C}_{6}\right)$ than women (36.0 vs $19.7 \%$, respectively) (Fig. 1).

When questioned by physicians, patients also reported suffering from visible signs of CVD (Table 2), most commonly telangiectases $(67.8 \%)$, varicose veins $(60.0 \%)$, and swollen legs (50.8\%). A high proportion of patients had suffered from symptoms in the last 4 weeks (Table 2).

The mean number of symptoms was $2.7 \pm 1.1$ (Table 2), with the majority of patients having more than one symptom: $13 \%$ suffered from one symptom, $24 \%$ had two symptoms, $28 \%$ had three symptoms, and $34 \%$ had four symptoms. Symptoms occurred mainly at the end of the day (72.8\%) and after prolonged standing (60.4\%). Mean symptom quantification on the VAS was $>5$ for all symptoms (Table 2), indicating a relatively high level of discomfort. Of note, the characteristics of patients excluded from the analysis, including disease severity, were similar to those of patients included in the analysis.

\section{Chronic venous disease treatment prescriptions at Vo}

At the V0 visit, 7352 patients $(99.4 \%)$ were prescribed VADs, mainly micronized purified flavonoid fraction (MPFF; 92.5\%) and diosmin (6.1\%), and 5003 patients (67.6\%) were prescribed compression therapy.

Prescription duration of VADs was $4-8$ weeks in $29.4 \%$ of patients, 9-12 weeks in $29.0 \%$ of patients, and $>12$ weeks in $37.2 \%$ of patients. About two-thirds $(66.2 \%)$ of patients were prescribed VADs for a duration of at least 8 weeks. MPFF prescription duration was $4-8$ weeks in $29.9 \%$ of patients, $9-12$ weeks in $29.5 \%$ of patients, and $>12$ weeks in $36.6 \%$ of patients.
Table 2 Chronic venous disease signs and symptoms in the study population at the inclusion visit (V0) $(n=7397)$

\begin{tabular}{|c|c|}
\hline Presence or severity of CVD signs and symptoms & Results \\
\hline \multicolumn{2}{|l|}{ Patient-reported signs ${ }^{\mathrm{a}}$ [no. of patients (\%)] } \\
\hline Telangiectases, reticular veins, spider veins & $5018(67.8)$ \\
\hline Varicose veins & $4435(60.0)$ \\
\hline Edema & $3760(50.8)$ \\
\hline Skin changes & $1346(18.2)$ \\
\hline Leg ulcer & $413(5.6)$ \\
\hline \multicolumn{2}{|l|}{ Patient-reported symptoms [no. of patients (\%)] } \\
\hline Heaviness, leg pain, sensation of swelling, cramps & $6629(89.6)$ \\
\hline \multicolumn{2}{|l|}{ Presence in the last 4 weeks of ${ }^{\mathrm{a}}$} \\
\hline Heaviness & $6289(85.0)$ \\
\hline Leg pain & $5583(75.5)$ \\
\hline Sensation of swelling & $5000(67.6)$ \\
\hline Cramps & $3626(49.0)$ \\
\hline Mean no. of symptoms \pm SD & $2.7 \pm 1.1$ \\
\hline \multicolumn{2}{|c|}{ Quantification of symptoms, in centimeters, on $\operatorname{VAS}^{\mathbf{a}(\text { mean } \pm \text { SD) }}$} \\
\hline Heaviness $(n=6246)$ & $5.7 \pm 2.1$ \\
\hline Leg pain $(n=5577)$ & $5.8 \pm 2.2$ \\
\hline Sensation of swelling $(n=4985)$ & $5.5 \pm 2.2$ \\
\hline Cramps $(n=3606)$ & $5.1 \pm 2.5$ \\
\hline \multicolumn{2}{|l|}{$\begin{array}{l}\text { Time when those leg problems were the most } \\
\left.\text { intense }^{\mathrm{a}} \text { [no. of patients }(\%)\right]\end{array}$} \\
\hline At the end of the day & $5383(72.8)$ \\
\hline After prolonged standing & $4470(60.4)$ \\
\hline During the night & $2271(30.7)$ \\
\hline \multicolumn{2}{|l|}{ Frequency of symptoms [no. of patients (\%)] } \\
\hline Never & $37(0.5)$ \\
\hline Rarely & $206(2.8)$ \\
\hline Occasionally & $1802(24.4)$ \\
\hline Regularly & $4630(62.6)$ \\
\hline All day and night & $673(9.1)$ \\
\hline Missing data & $49(0.7)$ \\
\hline
\end{tabular}

$C V D$ chronic venous disease, $S D$ standard deviation, $V A S$ visual analog scale

${ }^{\text {a }}$ Total of items can exceed total number of patients as patients can tick several boxes

Strength of compression therapy was mostly mild (15-21 mmHg) to moderate (23-32 $\mathrm{mmHg}$ ) [80.1\%]. Stockings were preferred over bandages in $78.2 \%$ of patients, and over two-thirds of patients (70.8\%) were prescribed compression therapy for at least 8 weeks. Prescription duration was $4-8$ weeks in $19.6 \%$ of patients, $9-12$ weeks in $17.5 \%$ of patients, and $>12$ weeks in $53.3 \%$ of patients.

The most frequently prescribed conservative therapeutic strategy consisted of a VAD + compression therapy (56.3\% of patients), followed by a VAD alone (33.4\%) (Fig. 2). Other treatment combinations included a VAD + compression + analgesics $(6.8 \%$ of patients), and a VAD + analgesics $(3.0 \%)$. Compression therapy and analgesics were 


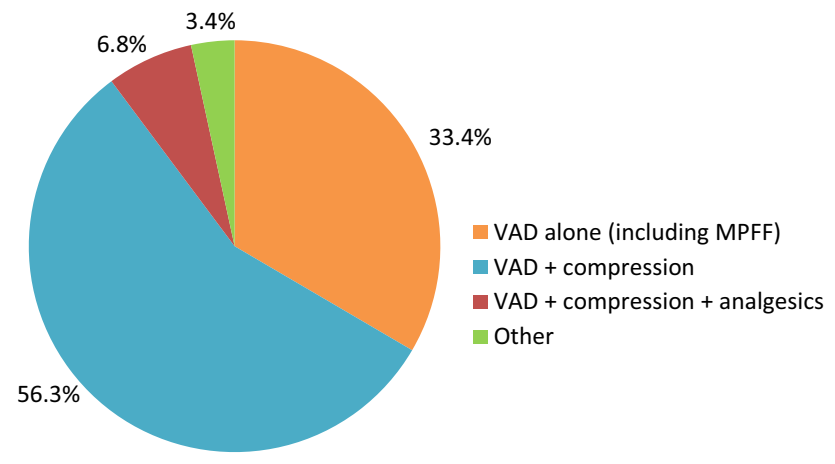

Fig. 2 Conservative therapy prescriptions for chronic venous disease at the inclusion visit (V0). MPFF micronized purified flavonoid fraction, $V A D$ venoactive drug

very rarely prescribed alone $(0.3$ and $<0.1 \%$ of patients, respectively).

A majority of patients (5793 patients; $78.3 \%$ ) was prescribed conservative treatment alone, $1598(21.6 \%)$ in association with operative treatment, and only $1(<0.1 \%)$ operative treatment alone.

\section{Influence of baseline characteristics on conservative treatment prescription}

VADs, including MPFF, were equally prescribed in men and women, but the prescription duration tended to be longer in men, with MPFF prescribed for $>12$ weeks in $41.5 \%$ of men compared with $35.7 \%$ of women. There was no relevant influence of gender on compression therapy prescription, except for a higher proportion of moderate or strong compression in men than in women (48.8 vs $35.5 \%$, respectively).

A VAD alone was most often prescribed in patients in the lower CEAP clinical classes. The proportion of patients prescribed a VAD alone decreased from $\mathrm{C}_{0}$ to $\mathrm{C}_{4}$ (53.9 and $24.0 \%$, respectively) and remained stable at $\approx 25 \%$ in classes $\mathrm{C}_{4 \mathrm{a}}-\mathrm{C}_{6}$. In contrast, the prescription of compression therapy $+\mathrm{VAD}$ increased with disease severity: compression + VAD was prescribed in 51-60\% of patients from CEAP clinical classes $\mathrm{C}_{1}-\mathrm{C}_{5}$ compared with $41 \%$ of $\mathrm{C}_{0 \mathrm{~s}}$ patients (Fig. 3). As might be expected, the prescription of analgesics increased with CVD severity (Fig. 3).

The prescribed duration of VAD treatment increased with disease severity. The proportion of patients prescribed VADs for $>12$ weeks was $21.6 \%$ in $\mathrm{C}_{0 \text { s }}$ patients, $33.4 \%$ in $\mathrm{C}_{1}-\mathrm{C}_{2}$ patients, and $39.6 \%$ in $\mathrm{C}_{3}-\mathrm{C}_{6}$ patients; the corresponding percentages for MPFF were 19.5, 32.9 and $38.4 \%$, respectively.

CEAP clinical class also influenced the prescribed strength and duration of compression therapy: severe CEAP

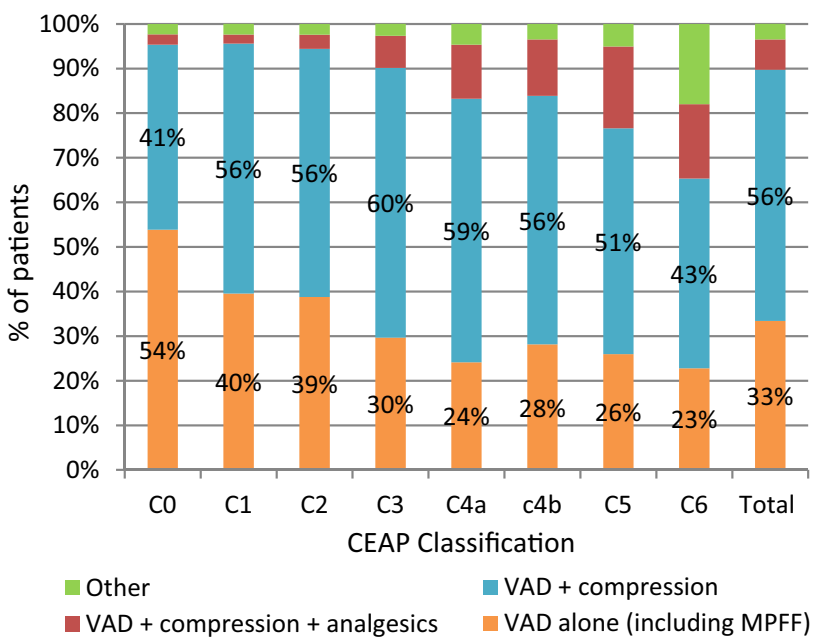

Fig. 3 Influence of CEAP class on type of conservative therapy prescribed. MPFF represented $93.0 \%$ of all prescriptions for a VAD. $C E A P$ Clinical Etiological Anatomic Pathophysiologic, MPFF micronized purified flavonoid fraction, $V A D$ venoactive drug

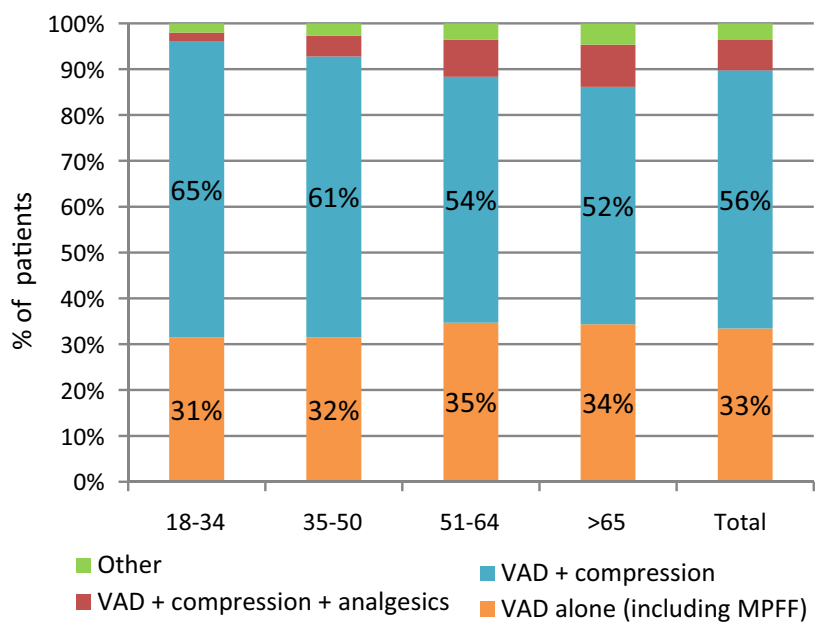

Fig. 4 Influence of age on type of conservative therapy prescribed. $M P F F$ micronized purified flavonoid fraction, $V A D$ venoactive drug

clinical classes were prescribed stronger compression than $\mathrm{C}_{0 \mathrm{~s}}$ and $\mathrm{C}_{1}$ patients. A prescription duration $>12$ weeks was noted in $59.2 \%$ of $\mathrm{C}_{5}-\mathrm{C}_{6}$ patients compared with $26.7 \%$ of $\mathrm{C}_{0 \mathrm{~s}}$ patients.

VADs were prescribed as monotherapy in approximately one-third of patients regardless of the age category, while the prescription of compression therapy + VAD decreased with increasing age, i.e. $65,61,54$, and $52 \%$ of patients in the $18-34,35-50,51-64$, and $>65$ years age classes, respectively (Fig. 4).

BMI category had no influence on VAD prescription as monotherapy. However, patients with a high BMI were less frequently prescribed compression $+\mathrm{VAD}$ than those with 
Table 3 Adherence to prescribed conservative therapy

\begin{tabular}{|llll|}
\hline Adherence & \multicolumn{2}{l}{ No. of patients (\%) } & \\
\cline { 2 - 4 } & $\begin{array}{l}\text { Venoactive } \\
\text { drugs (includ- } \\
\text { ing MPFF) } \\
(n=7352)\end{array}$ & $\begin{array}{l}\text { MPFF } \\
(n=6841)\end{array}$ & $\begin{array}{l}\text { Compression } \\
(n=5003)\end{array}$ \\
\hline $\begin{array}{l}\text { Adherent to treat- } \\
\text { ment/wearing } \\
\text { duration }\end{array}$ & $4797(65.2)$ & $4509(65.9)$ & $1458(29.1)$ \\
\hline $\begin{array}{l}\text { Adherent to dose/ } \\
\text { strength }\end{array}$ & $6385(86.8)$ & $6008(87.8)$ & $3988(79.7)$ \\
\hline
\end{tabular}

MPFF micronized purified flavonoid fraction

a lower BMI (e.g. $52.6 \%$ of patients with a BMI $\geq 30 \mathrm{~kg}$ / $\mathrm{m}^{2}$ compared with $63.8 \%$ in patients with a BMI $\leq 18 \mathrm{~kg} /$ $\mathrm{m}^{2}$ ), and more frequently prescribed analgesics + compression + VAD (9.2 vs $4.2 \%$, respectively).

\section{Treatment adherence}

Treatment adherence with VADs was high, with $65.2 \%$ of patients taking the treatment during the prescribed duration (persistent patients), and most patients (86.8\%) taking the prescribed dosage (Table 3). Of note, $7 \%$ of non-persistent patients took the treatment for a longer duration than prescribed, and $23 \%$ took the treatment for a shorter duration.

Nearly two-thirds $(65.9 \%)$ of patients were persistent to MPFF, with the majority (87.8\%) taking it at the prescribed dosage. Persistence to MPFF was particularly high (75.3\%) in patients prescribed MPFF for 2-8 weeks. There was a decrease in persistence with age, from $70.3 \%$ in the 18 - to 34-year range to $61.5 \%$ in patients aged $>65$ years, and with disease severity, from $67.4 \%$ in $\mathrm{C}_{0}-\mathrm{C}_{3}$ classes to $61.6 \%$ in $\mathrm{C}_{4}-\mathrm{C}_{6}$ classes. The main reason cited for non-persistence to MPFF was forgetfulness (26.5\%).

Patients were adherent to the prescribed strength of compression therapy (79.7\%), but only $29.1 \%$ of patients wore their compression as prescribed, with the remainder reporting that they wore it most days (27.7\%), intermittently $(22.6 \%)$ or not at all $(10.3 \%)$. In addition, only $64.5 \%$ of patients prescribed compression therapy returned for the follow-up visit wearing their compression hosiery. Persistence to compression therapy was similar in men and women and tended to decrease with age, from $34.5 \%$ in 18 - to 34 -yearolds to $27.0 \%$ in patients aged $>65$ years. The main reasons for non-persistence with compression therapy were related to discomfort with the product $(41.8 \%)$, difficulty in wearing $(40.1 \%)$, sweating $(20.5 \%)$, skin problems and itching $(19.8 \%)$, and unattractiveness (13.5\%).

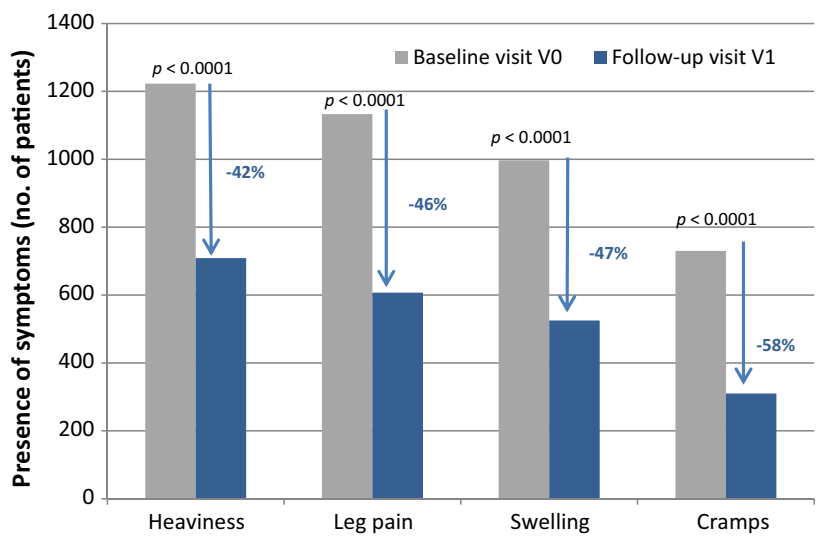

Fig. 5 Reduction in the number of patients with symptoms from the inclusion visit (V0) and the follow-up visit (V1) in patients receiving micronized purified flavonoid fraction only

\section{Treatment effectiveness and patient satisfaction}

Symptom relief was reported in $96.7 \%$ of patients treated with VADs only (96.5\% with MPFF) and $96.2 \%$ of patients treated with VAD + compression. Time to relief was $<3$ weeks for $71.5 \%$ of patients treated with a VAD only (71.8\% MPFF) and $60.3 \%$ of those treated with VAD+compression. There was no influence of baseline characteristics on the rate of patients with symptom relief and on time to relief.

In patients prescribed a VAD only, the presence of the most common leg symptoms was significantly reduced from $\mathrm{V} 0$ to $\mathrm{V} 1$, with a $42 \%$ reduction in the number of patients with leg heaviness, $47 \%$ reduction in those with leg pain, $48 \%$ reduction in those with leg swelling, and 57\% reduction in those with leg cramps. The corresponding reductions in patients prescribed MPFF were 42, 46, 47 and 58\%, respectively (all $p<0.0001$ ) [Fig. 5], and 33, 47, 41 and $57 \%$, respectively, in patients prescribed $\mathrm{VAD}+$ compression therapy combined.

Symptom intensity measured by VAS scores more than halved in patients treated with VADs, only from values of $6.0,6.2,5.8$ and $5.4 \mathrm{~cm}$ at $\mathrm{V} 0$ for leg heaviness, leg pain, sensation of swelling and cramps, respectively, to values of $2.9,2.8,2.7$ and $2.7 \mathrm{~cm}$, respectively, at V1. Mean reductions in VAS symptoms ranged from -2.8 to $-3.3 \mathrm{~cm}$ for a VAD only, -2.7 to $-3.0 \mathrm{~cm}$ for VAD + compression therapy, and -2.6 to $-3.5 \mathrm{~cm}$ for $\mathrm{VAD}+$ compression therapy + analgesics (Fig. 6).

The majority of patients were satisfied with their conservative treatment at V1 (94.1\%) and two-thirds of patients (66.0\%) were 'very' to 'extremely' satisfied. Similar results 


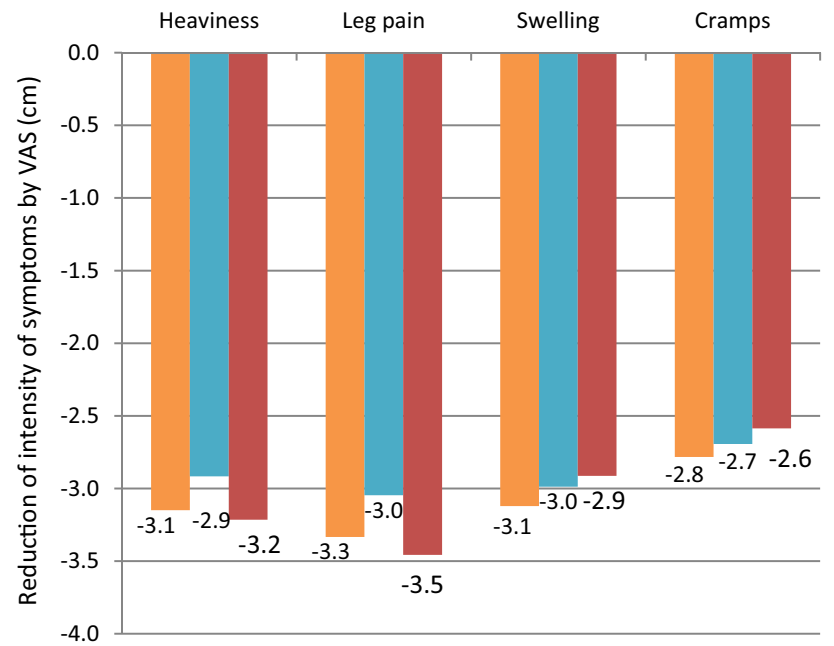

$\square$ VAD alone (including MPFF) $\quad$ VAD + compression $\square$ VAD + compression + analgesics

Fig. 6 Effect of various therapies in reducing the intensity of symptoms between the inclusion visit (V0) and the follow-up visit (V1), as measured by a $10-\mathrm{cm}$ VAS. $M P F F$ micronized purified flavonoid fraction, $V A D$ venoactive drug, $V A S$ visual analog scale

were obtained for patients receiving MPFF treatment. The median satisfaction score for both the combined conservative treatments and MPFF was 3.0, representing 'very satisfied'. The percentage of satisfied patients was very high across all CEAP clinical classes $(\approx 94 \%)$, except in the $\mathrm{C}_{0 \text { s }}$ class where it was slightly lower $(82.9 \%)$.

\section{Discussion}

The VAP program was specifically designed to provide a snapshot of current CVD management in routine practice and the beneficial effect of conservative treatments for patients across the spectrum of CVD and with varying levels of risk factors and symptom intensity. To the authors' knowledge this was the first worldwide, large-scale epidemiological survey since the Vein Consult Program was conducted between 2009 and 2011 [12], thus providing important, current data on a range of issues related to the management of CVD, including patient profiles, prescribed treatments, adherence and treatment effectiveness.

Nearly four times more women than men presented with CVD complaints (79.3 vs $20.7 \%$ ), although men represented a greater proportion of those in the more severe CVD classes. The highest proportion of subjects consulting for CVD was in the 51- to 64-year age group (34.8\%). CVD symptoms were reported by $89.6 \%$ of subjects and were present across all CEAP clinical classes. Intensity was high for all symptoms, even in the $\mathrm{C}_{0 \mathrm{~s}}$ class $(4.6-5.2 \mathrm{~cm}$ on VAS), particularly at the end of the day $(72.8 \%)$ and after prolonged standing (60.4\%). Despite the high prevalence of CVD signs and symptoms, only one-third of subjects (30.7\%) had previously consulted for leg problems and only one-quarter (24.3\%) had previously received treatment, confirming findings from other studies that CVD is underestimated by both patients and physicians, even when signs are present $[2,3$, 12]. Although venous symptoms are not specific for a diagnosis of CVD, certain characteristics should alert physicians to a CVD pathology. It has been shown that patients with CVD are significantly more likely to experience symptoms at the end of the day compared with other conditions that have similar symptoms, such as arthritis and peripheral arterial disease [13]. As with other chronic diseases, there is a need to identify patients earlier in the course of CVD, when effective conservative treatments can be used to prevent progression to more severe forms of the disease.

The majority of patients in the current study were prescribed conservative treatment $(78.3 \%)$, with only $21.6 \%$ undergoing operative treatment. Conservative therapy includes lifestyle advice in combination with compression therapy to decrease ambulatory venous hypertension and VADs to increase venous tone, and, in the case of MPFF, to prevent or inhibit the inflammatory response that contributes to disease progression. VADs, mainly MPFF, were a major component of conservative treatment in the current study across all age groups and all CEAP clinical classes, often in combination with compression therapy. MPFF is recommended by international guidelines for the reduction of CVD symptoms and as an adjunct to compression therapy for the healing of venous ulcers [14-16]. A prescription formulation of MPFF has also recently become available in the US [17]. Treatment was influenced by CEAP clinical class, with compression therapy more frequently forming part of the conservative therapy in patients with more severe complaints, and VAD alone in patients with less severe forms of CVD. Compression therapy was less frequently prescribed in older patients and in patients with high BMI, most probably due to difficulty in wearing in these particular groups of patients.

All conservative treatments were effective at reducing the presence, intensity and frequency of CVD symptoms from V0 to V1. Among patients with more severe CVD, the combination of a VAD with compression therapy was effective in spite of suboptimal compliance to compression therapy, with $30-40 \%$ of patients in CEAP clinical classes $\mathrm{C}_{4}-\mathrm{C}_{6}$ free of symptoms of leg heaviness, leg pain and sensation of swelling at V1, and $45-55 \%$ free of leg cramp.

Adherence with prescribed conservative treatment at V1 was highest for VADs, with $86.8 \%$ of patients reporting taking the dose as prescribed. In contrast, less than onethird of patients $(29.1 \%)$ wore their compression hosiery as prescribed. Compression therapy is very effective and is regarded as the gold-standard conservative treatment for CVD management in many countries, but poor adherence 
is well-documented $[18,19]$. In younger patients, issues with sweating and unattractiveness of the compression hosiery were responsible for most non-adherence, while in older patients and those with a high BMI, difficulty in putting the hosiery on and taking it off were the major factors. It should be noted that the section of the questionnaire related to compression therapy was subject to a large amount of missing data, suggesting a reluctancy of patients to answer. The true numbers of patients wearing their compression therapy as prescribed may therefore have been much lower.

The VAP is an important follow-up to the Vein Consult Program, an international, observational, prospective survey that collected global epidemiological data on CVD within the framework of ordinary consultations [12]. The Vein Consult Program analyzed data from 91,545 subjects, and a number of the demographic characteristics were similar to those in the VAP, including mean age of patients at consultation and mean BMI value; however, some differences are also worth noting. In the current study, CEAP clinical classes $\mathrm{C}_{0 \mathrm{~s}}-\mathrm{C}_{2}$ accounted for $44 \%$ of subjects, only $3.5 \%$ of whom were $\mathrm{C}_{0 \mathrm{~s}}$ subjects, whereas in the Vein Consult Program, $\mathrm{C}_{0 \mathrm{~s}}-\mathrm{C}_{2}$ made up $60 \%$ of the study population, with a particularly high proportion of $\mathrm{C}_{0 \mathrm{~s}}$ subjects (20\%) [12]. The more severe CEAP clinical classes $\left(\mathrm{C}_{3}-\mathrm{C}_{6}\right)$ made up over half (55\%) of the VAP population, but only one-quarter (24.6\%) of the Vein Consult Program. Potential reasons for the greater predominance of the more severe CEAP clinical classes in the current study compared with the Vein Consult Program may be related to differences in the distribution of patients across age categories, and to variations in risk factors associated with disease severity between the two populations. It is also possible that the participating primary care physicians in the VAP were less informed about the manifestations in the early stages. That CVD is often overlooked by physicians was recently demonstrated in a Belgian epidemiological study where only 7.8 and $40.5 \%$ of $\mathrm{C}_{0 \mathrm{~s}}$ and $\mathrm{C}_{1}$ patients, respectively, were considered by their physicians to have CVD, with actual figures rising to more than $80 \%$ from $\mathrm{C}_{2}$ and up to $94.3 \%$ at $\mathrm{C}_{5}$ [3]. An interpretation of these results is that until the occurrence of varicose veins, physicians do not consider patients as suffering from CVD.

CVD is related to primary failure of venous valves that are affected by inflammation [20,21]. As a result, a number of guidelines and systematic reviews now recognize that early pharmacological treatment directed towards preventing or inhibiting the inflammatory response at all stages of the disease may play a significant role in preventing or slowing the development and recurrence of CVD signs and symptoms [14, 17, 22-25]. Unlike surgical interventions, conservative treatment, including VADs, cannot restore varicose veins; however, they have significant potential for slowing or preventing progression of CVD in the earlier stages, and for the relief of symptoms throughout the disease process.

This observational survey was subject to certain limitations, namely the possibility for introduction of sample bias based on how representative the study sample was compared with the general population with CVD, and whether management practices at participating sites reflected those practiced at a national level. Further confounders include the possibility of incomplete survey response data, as well as the potential inaccuracy of self-reported behavior. In addition, the VAP was limited to a clinical examination, and the patient's medical history did not include additional methods such as Doppler analysis. The VAP only collected data from subjects consulting spontaneously for CVD-related complaints in routine clinical practice, excluding those receiving ongoing treatment for CVD or those consulting for a CVDrelated emergency. The demographic data and risk factor profile of the study population was consistent with that of the much larger Vein Consult Program [12], suggesting that the population was representative of patients routinely seen in clinical practice. The results therefore provide valuable upto-date information on the characteristics of patients presenting with CVD-related complaints, the range and severity of complaints, and their current management worldwide. The challenge is to alert physicians to the need for early recognition of the signs and symptoms and to promote early treatment to offset the progression of the disease and improve QoL. This may involve education programs and the availability of patient brochures and other materials, as well as family screening, to encourage identification of patients at $\mathrm{C}_{1}$ and $\mathrm{C}_{2}$ CEAP clinical stages.

\section{Conclusion}

The VAP provided large-scale data on the management of CVD focusing on the prevalence of symptoms after treatment initiation, treatment effectiveness, adherence and satisfaction with conservative treatment in the framework of routine consultations, while examining the burden of CVD among populations in different regions of the world. The majority of patients were treated with conservative therapy, including lifestyle advice, VADs and compression therapy. VADs, and in particular MPFF, represented the major component of conservative therapy, with compression added more frequently in more severe patients and VADs prescribed alone in less severe cases. All methods of conservative therapy were effective at reducing CVD symptoms. Adherence to therapy was highest for VADs, mainly in the form of MPFF. This survey confirms the effectiveness and very high levels of patient satisfaction with conservative treatments across the spectrum of CVD. The findings should encourage physicians to diagnose 
patients and initiate treatment at earlier stages of the disease to prevent any disease worsening and to preserve patients' QoL, avoid or delay any invasive treatments, and thus reduce healthcare costs.

Acknowledgements The authors would like to express their thanks to all the physicians and their patients who participated in this study.

\section{Compliance with ethical standards}

Ethical approval Approval confirming compliance with the ethical standards laid down in the Helsinki Declaration amended in October 2008 was obtained from the Ethics Committees of all countries in which the program was implemented.

Informed consent Informed consent was obtained from each participant in this study.

Conflict of interest Vadim Bogachev, José Manuel Jiménez Arribas, Sorin Baila, Jorge Ulloa Dominguez, Johannes Walter, Dale Maharaj, and Andres Marin report no conflicts of interest associated with this study.

Editorial assistance and funding Editorial assistance for this paper was provided by Jenny Grice and funded by Servier Affaires Médicales.

Open Access This article is distributed under the terms of the Creative Commons Attribution-NonCommercial 4.0 International License (http://creativecommons.org/licenses/by-nc/4.0/), which permits any noncommercial use, distribution, and reproduction in any medium, provided you give appropriate credit to the original author(s) and the source, provide a link to the Creative Commons license, and indicate if changes were made.

\section{References}

1. Beebe-Dimmer JL, Pfeifer JR, Engle JS, et al. The epidemiology of chronic venous insufficiency and varicose veins. Ann Epidemiol. 2005;15(3): 175-84.

2. McLafferty RB, Passman MA, Caprini JA, et al. Increasing awareness about venous disease: the American Venous Forum expands the National Venous Screening Program. J Vasc Surg. 2008;48(2):394-9.

3. Vuylsteke ME, Thomis S, Guillaume G, et al. Epidemiological study on chronic venous disease in Belgium and Luxembourg: prevalence, risk factors, and symptomatology. Eur J Vasc Endovasc Surg. 2015;49(4):432-9.

4. Eklof B, Rutherford RB, Bergan JJ, et al. American Venous Forum International Ad Hoc Committee for Revision of the CEAP Classification. Revision of the CEAP classification for chronic venous disorders: consensus statement. J Vasc Surg. 2004;40:1248-52.

5. Lattimer CR. CVD: a condition of underestimated severity. Int Angiol. 2014;33(3):222-8.

6. Wittens C, Davies AH, Bækgaard N, et al. Editor's choice-management of chronic venous disease: clinical practice guidelines of the European Society for Vascular Surgery (ESVS). Eur J Vasc Endovasc Surg. 2015;49(6):678-737.

7. Onida S, Davies AH. Predicted burden of venous disease. Phlebology. 2016;31(1 Suppl):74-9.
8. Bergan JJ, Schmid-Schönbein GW, Coleridge Smith PD, et al. Chronic venous disease. N Engl J Med. 2006;355:488-98.

9. Aloi TL, Camporese G, Izzo M, et al. Refining diagnosis and management of chronic venous disease: outcomes of a modified Delphi consensus process. Eur J Intern Med. 2019. https://doi. org/10.1016/j.ejim.2019.03.005 (Epub 18 Mar 2019).

10. Mansilha A, Sousa J. Pathophysiological mechanisms of chronic venous disease and implications for venoactive drug therapy. Int J Mol Sci. 2018;19(6):E1669.

11. Eklof B, Perrin M, Delis K, et al. Updated terminology of chronic venous disorders: the vein term transatlantic interdisciplinary consensus document. J Vasc Surg. 2009;49:498-501.

12. Rabe E, Guex JJ, Puskas A, et al. VCP Coordinators. Epidemiology of chronic venous disorders in geographically diverse populations: results from the Vein Consult Program. Int Angiol. 2012;31(2):105-15

13. Van der Velden SK, Shadid NH, Nelemans PJ, et al. How specific are venous symptoms for diagnosis of chronic venous disease? Phlebology. 2014;29(9):580-6.

14. Nicolaides A, Kakkos S, Baekgaard N, et al. Management of chronic venous disorders of the lower limbs. Guidelines according to scientific evidence. Part I. Int Angiol. 2018;37(3):181-254.

15. Nicolaides A, Kakkos S, Eklof B, et al. Management of chronic venous disorders of the lower limbs. Guidelines according to scientific evidence. Int Angiol. 2014;33(2):126-39.

16. Gloviczki P, Comerota AJ, Dalsing MC, et al. Society for Vascular Surgery; American Venous Forum. The care of patients with varicose veins and associated chronic venous diseases: clinical practice guidelines of the Society for Vascular Surgery and the American Venous Forum. J Vasc Surg. 2011;53(5 Suppl):2S-48S.

17. Bush R, Comerota A, Meissner M, et al. Recommendations for the medical management of chronic venous disease: the role of micronized purified flavanoid fraction (MPFF). Phlebology. 2017;32(1 Suppl):3-19.

18. Raju S, Hollis K, Neglen P. Use of compression stockings in chronic venous disease: patient compliance and efficacy. Ann Vasc Surg. 2007;21(6):790-5.

19. Moffatt C, Kommala D, Dourdin N, et al. Venous leg ulcers: patient concordance with compression therapy and its impact on healing and prevention of recurrence. Int Wound J. 2009;6(5):386-93.

20. Takase S, Pascarella L, Lerond L, et al. Venous hypertension, inflammation and valve remodeling. Eur J Vasc Endovasc Surg. 2004;28(5):484-93.

21. Pascarella L, Lulic D, Penn AH, et al. Mechanisms in experimental venous valve failure and their modification by Daflon $500 \mathrm{mg}$. Eur J Vasc Endovasc Surg. 2008;35(1):102-10.

22. Nicolaides AN, Allegra C, Bergan J, et al. Management of chronic venous disorders of the lower limbs: guidelines according to scientific evidence. Int Angiol. 2008;27:1-59.

23. Kearon C, Kahn SR, Agnelli G, et al. American College of Chest Physicians. Antithrombotic therapy for venous thromboembolic disease: American College of Chest Physicians Evidence-Based Clinical Practice Guidelines (8th Edition). Chest. 2008;133(6 Suppl):454S-545S.

24. Coleridge Smith PD. Drug treatment of varicose veins, venous oedema, and ulcers. In: Gloviczki P, editor. Handbook of venous disorders: guidelines of the American venous Forum. 3rd ed. London: Hodder Arnold; 2009. p. 359-65.

25. Bogachev VY, Boldin BV, Lobanov VN. Benefits of micronized purified flavonoid fraction as adjuvant therapy on the inflammatory response after sclerotherapy. Int Angiol. 2018;37(1):71-8. 